C by R. Oldenbourg Verlag, München 1997

\title{
Crystal structure of 10-( $3^{\prime}-N$-morpholinopropyl)-2-trifluoro phenoxazine hydrochloride, $\mathrm{C}_{20} \mathrm{H}_{21} \mathrm{ClF}_{3} \mathrm{~N}_{2} \mathrm{O}_{2}$
}

\author{
N. K. Lokanath, M. A. Sridhar, J. Shashidhara Prasad
}

University of Mysore. Department of Studies in Physics. Manasagangothri. Mysore 570 006. India

\section{Ramegowda}

Lecturer in Physics, Government College for Boys, Mandya 571 401, India

G. B. Eregowda and K. N. Thimmaiah

University of Mysore, Department of Studies in Chemistry, Manasagangothri, Mysore 570 006, India

Received May 28, 1996, CSD-No. 402510

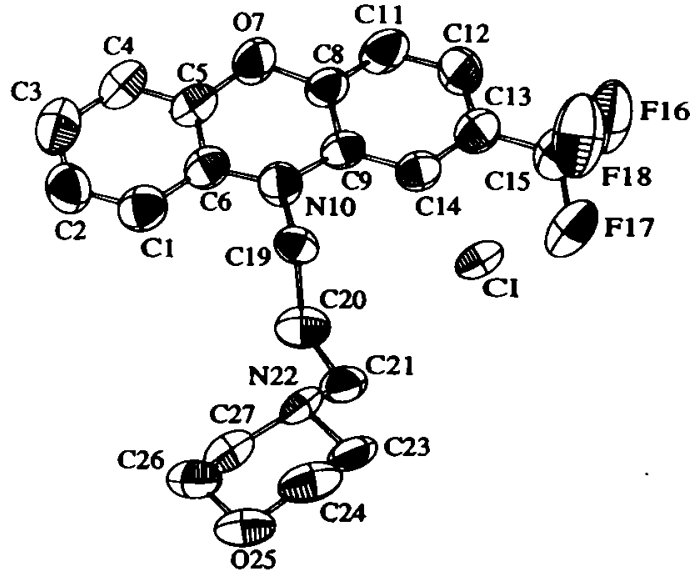

Fig. 1. ORTEP plot of the molecules.

Table 1. Parameters used for the X-ray data collection

\begin{tabular}{ll}
\hline Crystal: & $\begin{array}{l}\text { transparent, rectangular solid, } \\
\text { size } 0.1 \times 0.2 \times 0.2 \mathrm{~mm}\end{array}$ \\
Wavelength: & Mo $K_{\alpha}$ radiation $(0.71069 \AA)$ \\
$\mu:$ & $2.40 \mathrm{~cm}^{-1}$ \\
Diffractometer: & Rigaku AFC7S \\
Scan mode: & $\omega / 2 \theta$ \\
$T_{\text {measurement: }}$ & $293 \mathrm{~K}$ \\
$2 \theta_{\text {max }}$ & $50^{\circ}$ \\
N(hkl) & 3467 \\
Criterion for $I_{0}:$ & $I_{0}>2 \sigma\left(I_{0}\right)$ \\
N(param)refined: & 254 \\
Programs: & SHELXS-86, SHELXL-93 \\
\hline
\end{tabular}

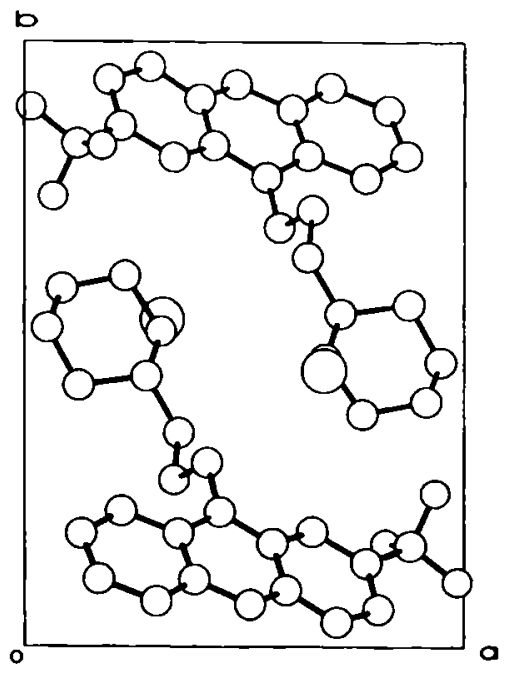

Fig. 2. Packing of the molecules down $c$.

Source of material: synthesized (see ref. 1).

The bond distances and angles do not show any large deviations. The thermal ellipsoid plot of the molecule is shown in Fig. 1. The packing of the molecules down $c$ is shown in Fig. 2. The molecules are stacked in two layers: one layer has the three fused rings with the fluorine atoms and the other layer has the cyclohexane ring and the free chlorine.

$\mathrm{C}_{20} \mathrm{H}_{21} \mathrm{ClF}_{3} \mathrm{~N}_{2} \mathrm{O}_{2}$, triclinic, $P \overline{1}$ (No. 2), $a=9.7757(9) \AA$, $b=13.826(1) \AA, c=7.4117(9) \AA, \alpha=99.098(9)^{\circ}, \beta=95.249(9)^{\circ}$, $\gamma=89.740(9)^{\circ}, V=984.9 \AA^{3}, Z=2, R(F)=0.090, R_{\mathrm{w}}\left(F^{2}\right)=0.284$.

Table 2. Final atomic coordinates and displacement parameters (in $\AA^{2}$ )

\begin{tabular}{|c|c|c|c|c|c|c|c|c|c|c|}
\hline Atom & Site & $x$ & $y$ & $z$ & $U_{11}$ & $U_{22}$ & $U_{33}$ & $U_{12}$ & $U_{13}$ & $U_{23}$ \\
\hline$C(1)$ & $2 i$ & $0.7805(9)$ & $0.7756(6)$ & $-0.210(1)$ & $0.077(5)$ & $0.065(4)$ & $0.058(4)$ & $0.004(4)$ & $0.004(4)$ & $0.004(3)$ \\
\hline$C(2)$ & $2 i$ & $0.8714(9)$ & $0.8128(7)$ & $-0.316(1)$ & $0.060(4)$ & $0.088(6)$ & $0.069(5)$ & $0.006(4)$ & $0.010(4)$ & $-0.002(4)$ \\
\hline$C(3)$ & $2 i$ & $0.831(1)$ & $0.8880(7)$ & $-0.411(1)$ & $0.083(6)$ & $0.084(6)$ & $0.071(5)$ & $-0.017(5)$ & $0.023(4)$ & $0.003(4)$ \\
\hline$C(4)$ & $2 i$ & $0.700(1)$ & $0.9252(6)$ & $-0.399(1)$ & $0.093(6)$ & $0.075(5)$ & $0.051(4)$ & $0.000(4)$ & $0.018(4)$ & $0.016(4)$ \\
\hline$C(5)$ & $2 i$ & $0.6121(8)$ & $0.8888(5)$ & $-0.296(1)$ & $0.080(5)$ & $0.058(4)$ & $0.045(4)$ & $0.004(3)$ & $0.008(3)$ & $0.010(3)$ \\
\hline
\end{tabular}


Table 2. (Continued)

\begin{tabular}{|c|c|c|c|c|c|c|c|c|c|c|}
\hline Atom & Site & $x$ & $y$ & $z$ & $U_{11}$ & $U_{22}$ & $U_{33}$ & $U_{12}$ & $U_{13}$ & $U_{23}$ \\
\hline$C(6)$ & $2 i$ & $0.6490(8)$ & $0.8127(5)$ & $-0.1970(9)$ & $0.068(4)$ & $0.049(3)$ & $0.047(3)$ & $0.002(3)$ & $0.006(3)$ & $0.003(3)$ \\
\hline$O(7)$ & $2 i$ & $0.4840(6)$ & $0.9302(4)$ & $-0.2909(8)$ & $0.072(3)$ & $0.086(4)$ & $0.077(4)$ & $0.018(3)$ & $0.021(3)$ & $0.048(3)$ \\
\hline$C(8)$ & $2 i$ & $0.4008(7)$ & $0.9066(5)$ & $-0.1649(9)$ & $0.064(4)$ & $0.055(4)$ & $0.049(4)$ & $0.008(3)$ & $0.006(3)$ & $0.021(3)$ \\
\hline$C(9)$ & $2 i$ & $0.4325(7)$ & $0.8294(5)$ & $-0.0638(9)$ & $0.064(4)$ & $0.044(3)$ & $0.045(3)$ & $0.002(3)$ & $0.001(3)$ & $0.012(3)$ \\
\hline$N(10)$ & $2 i$ & $0.5540(6)$ & $0.7769(4)$ & $-0.0970(9)$ & $0.059(3)$ & $0.058(3)$ & $0.075(4)$ & $0.012(3)$ & $0.009(3)$ & $0.031(3)$ \\
\hline$C(11)$ & $2 i$ & $0.2853(9)$ & $0.9594(6)$ & $-0.143(1)$ & $0.088(5)$ & $0.066(5)$ & $0.063(4)$ & $0.017(4)$ & $0.014(4)$ & $0.031(4)$ \\
\hline$C(12)$ & $2 i$ & $0.1917(8)$ & $0.9380(6)$ & $-0.026(1)$ & $0.064(4)$ & $0.070(5)$ & $0.075(5)$ & $0.027(4)$ & $0.016(4)$ & $0.020(4)$ \\
\hline$C(13)$ & $2 i$ & $0.2188(8)$ & $0.8614(5)$ & $0.073(1)$ & $0.071(4)$ & $0.055(4)$ & $0.055(4)$ & $-0.002(3)$ & $0.007(3)$ & $0.009(3)$ \\
\hline$C(14)$ & $2 i$ & $0.3410(7)$ & $0.8098(5)$ & $0.0552(9)$ & $0.063(4)$ & $0.048(3)$ & $0.051(4)$ & $0.001(3)$ & $0.001(3)$ & $0.014(3)$ \\
\hline$C(15)$ & $2 i$ & $0.119(1)$ & $0.8327(7)$ & $0.195(1)$ & $0.095(6)$ & $0.071(5)$ & $0.077(6)$ & $0.008(5)$ & $0.022(5)$ & $0.017(4)$ \\
\hline$F(16)$ & $2 i$ & $0.0146(7)$ & $0.8926(5)$ & $0.214(1)$ & $0.117(5)$ & $0.113(5)$ & $0.141(6)$ & $0.032(4)$ & $0.074(4)$ & $0.048(4)$ \\
\hline $\mathbf{F}(17)$ & $2 i$ & $0.1756(7)$ & $0.8291(7)$ & $0.3618(9)$ & $0.122(5)$ & $0.198(8)$ & $0.078(4)$ & $-0.015(5)$ & $0.028(3)$ & $0.058(4)$ \\
\hline$F(18)$ & $2 i$ & $0.0645(8)$ & $0.7453(5)$ & $0.134(1)$ & $0.140(6)$ & $0.081(4)$ & $0.167(7)$ & $-0.029(4)$ & $0.071(5)$ & $0.011(4)$ \\
\hline$C(19)$ & $2 i$ & $0.5832(7)$ & $0.6940(5)$ & $-0.0004(9)$ & $0.050(3)$ & $0.048(3)$ & $0.059(4)$ & $0.006(3)$ & $-0.005(3)$ & $0.015(3)$ \\
\hline$N(22)$ & $2 i$ & $0.7232(6)$ & $0.5518(4)$ & $0.2523(6)$ & $0.073(4)$ & $0.054(3)$ & $0.027(2)$ & $-0.009(2)$ & $0.006(2)$ & $0.011(2)$ \\
\hline$C(23)$ & $2 i$ & $0.6890(8)$ & $0.4773(6)$ & $0.368(1)$ & $0.079(5)$ & $0.086(5)$ & $0.040(3)$ & $-0.015(4)$ & $-0.007(3)$ & $0.031(3)$ \\
\hline$C(24)$ & $2 i$ & $0.773(1)$ & $0.3860(6)$ & $0.326(1)$ & $0.125(8)$ & $0.064(5)$ & $0.061(5)$ & $-0.010(5)$ & $-0.020(5)$ & $0.028(4)$ \\
\hline$C(26)$ & $2 i$ & $0.9482(9)$ & $0.4717(7)$ & $0.232(1)$ & $0.079(5)$ & $0.085(6)$ & $0.055(4)$ & $0.009(4)$ & $-0.015(4)$ & $0.011(4)$ \\
\hline$C(27)$ & $2 i$ & $0.8774(9)$ & $0.5671(6)$ & $0.270(1)$ & $0.094(6)$ & $0.063(4)$ & $0.041(3)$ & $-0.005(4)$ & $0.005(3)$ & $0.014(3)$ \\
\hline$O(25)$ & $2 i$ & $0.9159(7)$ & $0.4066(4)$ & $0.3530(8)$ & $0.101(4)$ & $0.070(3)$ & $0.061(3)$ & $0.014(3)$ & $-0.020(3)$ & $0.022(3)$ \\
\hline $\mathrm{Cl}$ & $2 i$ & $0.3142(2)$ & $0.5425(1)$ & $0.1570(2)$ & $0.082(1)$ & $0.058(1)$ & $0.0326(9)$ & $-0.0014(8)$ & $0.0021(7)$ & $0.0085(7)$ \\
\hline
\end{tabular}

Acknowledgment. The authors would like to express their thanks to DST, Government of India for financial assistance under the project SP/12/FOO/93.

\section{References}

1. Dass, C.; Thimmaiah, K. N.: Jayashree, B. S.; Seshadri, R.; Israel, M.; Houghton, P. J.: Analysis of phenoxazine chemosensitizers: An electron ionization and $\mathrm{keV}$-ion beam bombardment mass spectrometry study. Biol. Mass. Spectrometry 23 (1994) 140-146.

2. Sheldrick, G. M.: Phase annealing in SHELX-90: Direct methods for larger structures. Acta Crystallogr. A46 (1990) 467-473.

3. Sheldrick, G. M.: SHELXL-93. Crystal structure refinement program. University of Göttingen, Germany 1993. 